Copyright by the American Physical Society. Li, M. C., "Direct phase determination in Crystallography," Phys. Rev. B 14, 5529 DOI: http://dx.doi.org/10.1103/PhysRevB.14.5529

\title{
Direct phase determination in crystallography
}

\author{
Ming Chiang Li \\ Department of Physics, Virginia Polytechnic Institute and State University, Blacksburg, Virginia 24061
}

(Received 10 June 1976)

\begin{abstract}
It was shown theoretically that through the diffraction of two coherent beams, one is able to measure both the magnitude and phase of the structure factor directly. Here some experimental comments concerning the performance of the proposed experiment are presented.
\end{abstract}

In several previous papers, ${ }^{1}$ the crystal diffraction of two coherent beams was studied. It was shown that through the diffraction of two coherent beams, one is able to measure both the magnitude and phase of the structure factor directly. Here I wish to present some experimental comments concerning the performance of the above proposed experiment.

In the crystal diffraction process, the incident beams are described by the wave function

$$
e^{i \overrightarrow{\mathbf{r}}_{1} \cdot \overrightarrow{\mathbf{r}}}+a e^{i \overrightarrow{\mathbf{k}}_{2} \cdot \overrightarrow{\mathbf{r}}},
$$

where $a$ denotes the relative phase and amplitude of these two waves. The wave vectors satisfy relations

$$
\begin{gathered}
\overrightarrow{\mathrm{k}}_{1}^{2}=\overrightarrow{\mathrm{k}}_{2}^{2}=\overrightarrow{\mathrm{k}}^{2} \\
\overrightarrow{\mathrm{k}}_{1}-\overrightarrow{\mathrm{k}}_{2}=\vec{\kappa},
\end{gathered}
$$

and

where $\vec{k}$ is the wave vector of a diffracted beam and $\vec{k}$ is a reciprocal-lattice vector of the crystal. The experimentally measured intensity can be expressed as

$$
I\left(\overrightarrow{\mathrm{k}}_{1}, \overrightarrow{\mathrm{k}}_{2} ; \overrightarrow{\mathrm{k}}\right)=|G|^{2}|E|^{2},
$$

where

$$
E=f\left(\overrightarrow{\mathrm{k}}_{1} ; \overrightarrow{\mathrm{k}}\right)+a f\left(\overrightarrow{\mathrm{k}}_{2} ; \overrightarrow{\mathrm{k}}\right)
$$

and

$$
G=\sum_{i=1}^{N} e^{-i\left(\overrightarrow{\mathrm{k}}-\overrightarrow{\mathrm{k}}_{1}\right) \cdot \overrightarrow{\mathrm{r}}_{i}}=\sum_{i=1}^{N} e^{-i\left(\overrightarrow{\mathrm{k}}-\overrightarrow{\mathrm{k}}_{2}\right) \cdot \overrightarrow{\mathrm{r}}_{i}} .
$$

$\vec{r}_{i}$ is the position vector of a unit cell and $N$ is the total number of unit cells in the scattering region. In crystal diffraction, $E$ is called the structure factor and $G$ the lattice factor, which depends on the type of lattice. $f\left(\overrightarrow{\mathrm{k}}_{l} ; \overrightarrow{\mathrm{k}}\right)$ for $l=1,2$ is the structure factor in the crystal diffraction of a single incident beam. It is well known that in conventional diffraction process the magnitude of the structure factor $f\left(\overrightarrow{\mathrm{k}}_{l} ; \overrightarrow{\mathrm{k}}\right)$ is the only experimentally measur able quantity. From Eqs. (3) and (4), it is easy to see that the diffraction of two coherent beams provides the experimental information on the term

$$
\operatorname{Re}\left[a f\left(\overrightarrow{\mathrm{k}}_{2} ; \overrightarrow{\mathrm{k}}\right) f^{*}\left(\overrightarrow{\mathrm{k}}_{1} ; \overrightarrow{\mathrm{k}}\right)\right],
$$

from which one can deduce directly the phase of the structure factor $f\left(\overrightarrow{\mathrm{k}}_{l} ; \overrightarrow{\mathrm{k}}\right) .^{2}$

To initiate the crystal diffraction of two coherent beams, the wave-vector difference between the beams should be equal to a reciprocal-lattice vector of the crystal as expressed by Eq. (3). This is a stringent requirement. The main interest of the present comments is on how to fulfill such a requirement.

There have been successful experiments on the crystal diffraction by two coherent beams. The diffraction of two coherent electron beams was carried out by Marton, Simpson, and Suddeth in the Marton-type electron interferometer. ${ }^{3}$ The diffraction of two coherent $x$-ray beams was performed by Bonse and Hart. ${ }^{4}$ Recently, the diffraction of two coherent neutron beams was reported by Rauch, Treimer, and Bonse. ${ }^{5}$ None of these experimenters had ever attempted to measure the phase of the structure factor $f\left(\overrightarrow{\mathrm{k}}_{l} ; \overrightarrow{\mathbf{k}}\right)$. In all those experiments three identical single crystals were used. The first one is a splitter. This is an amplitude division of two coherent beams. After the selection two diffracted beams are bent by the second crystal. In the intersection of two bent beams, the third crystal is placed. The crystal diffraction of two coherent beams is occurred in the third crystal. Since all crystals are identical, there is no problem to satisfy Eq. (3). If the above suggested arrangement is adopted, the proposed experiment is easy to perform. For the crystal diffraction of two coherent electron beams, one needs simply to repeat the Marton, Simpson, and Suddeth experiment with the electron detector replacing the electron microscope. For crystal diffraction of two coherent $x$-ray or neutron beams, one repeats the experiments performed by Bonse et al., preferably with single crystals reoriented in such a way that there are more than two diffracted beams in the third crystal diffraction.

Suppose it is difficult to prepare a large singlecrystal sample for study; then the sample cannot 
be used as a beam bender. Alternatively, we propose the experiment of using four single crystals. Three of them are perfect crystals. The first crystal is still used as the beam splitter. After splitting two diffracted beams with wave vectors $\overrightarrow{\mathrm{K}}_{1}$ and $\overrightarrow{\mathrm{K}}_{2}$ are selected out. Then these two beams are bent separately by the next two crystals with the reciprocal-lattice vectors $\vec{\kappa}_{b}^{1}$ and $\vec{\kappa}_{b}^{2}$. The fourth crystal is the sample and is placed at the intersection of these bent beams. The incident wave vectors at the sample can be expressed as

$$
\overrightarrow{\mathrm{k}}_{1}=\overrightarrow{\mathrm{K}}_{1}-\vec{\kappa}_{b}^{1}
$$

and

$$
\overrightarrow{\mathrm{k}}_{2}=\overrightarrow{\mathrm{K}}_{2}-\vec{\kappa}_{b}^{2} \text {. }
$$

To initiate the two coherent beam diffractions, the difference of these two vectors should be equal to a reciprocal-lattice vector $\vec{\kappa}$ of the sample. That is to say

$$
\vec{\kappa}=\overrightarrow{\mathrm{k}}_{1}-\overrightarrow{\mathrm{k}}_{2}=\vec{\kappa}_{s}-\vec{\kappa}_{b}^{1}+\vec{\kappa}_{b}^{2},
$$

where

$$
\vec{\kappa}_{s}=\overrightarrow{\mathrm{K}}_{1}-\overrightarrow{\mathrm{K}}_{2}
$$

is a reciprocal-lattice vector of the beam splitter. The experimental procedure is to align these four crystals such that Eq. (10) is satisfied. To see how to achieve alignment let us consider a simple example. If vectors $\vec{\kappa}$ and $\vec{\kappa}_{s}$ are in the direction $\overrightarrow{\mathrm{n}}$ and parallel, then

$$
\vec{\kappa}-\vec{\kappa}_{s}=\left(\kappa-\kappa_{s}\right) \overrightarrow{\mathrm{n}} \text {. }
$$

Furthermore, two benders are identical with

$$
\vec{\kappa}_{b}^{1}=\kappa_{b} \vec{n}_{1}
$$

and

$$
\vec{\kappa}_{b}^{2}=\kappa_{b} \overrightarrow{\mathrm{n}}_{2},
$$

where $\vec{n}_{1}$ and $\vec{n}_{2}$ are the unit vectors. From the above choice, Eq. (10) can be rewritten

$$
\overrightarrow{\mathrm{n}}_{1} \cdot \overrightarrow{\mathrm{n}}_{2}=\left[2 \kappa_{b}^{2}-\left(\kappa-\kappa_{s}\right)^{2}\right] / 2 \kappa_{b}^{2}
$$

and

$$
\overrightarrow{\mathrm{n}} \cdot \overrightarrow{\mathrm{n}}_{2}=\left(\kappa-\kappa_{s}\right) / 2 \kappa_{b} .
$$

Then the alignment procedure satisfies Eqs. (12), (15), and (16). The only difficult part of the proposed experiment is to align these four crystals. However, Marton, Simpson, and Suddeth ${ }^{3}$ were able to align three separate crystals to success- fully perform the electron interference experiment, for which the electron has only a wavelength $0.04 \AA$.

The techniques of systematic bending and twisting are widely practiced in the experimentation of crystal diffractions. ${ }^{6}$ Through a shearing deformation one can change the lattice spacing or the magnitude of a reciprocal vector. Another method is to choose a giant-size perfect crystal with the reciprocal vector nearly equal to that of the sample crystal. From the giant crystal one cuts out a beam splitter and a beam bender. With the help of shearing deformation, one can bring the reciprocal vectors of the splitter and bender equal to that of the sample. By doing this one retains the threecrystal configuration, which was used by the experimenters in the early two coherent beam diffraction. ${ }^{3-5}$

The electron density of a crystal inside a unit cell is the Fourier transform of the structure factor $f\left(\overrightarrow{\mathrm{k}}_{l} ; \overrightarrow{\mathrm{k}}\right)$. The lack of a direct experimental phase information of the structure factor reveals that the electron density cannot be deduced directly from the experiment. This constitutes the famous phase problem of crystallography. In the absence of direct phase information, crystal structures are determined in an indirect way. Even so, not every crystal structure can be solved by methods currently known.?

The current views on the phase problem are interesting. Two opposite views are quoted here. In the first view ${ }^{8}$ it is said that for the most experimental situations complete knowledge of the electron density is neither necessary nor desirable, since it would involve knowledge of the relative positions and bonding of all the atoms of the sample and represent a quantity of data which would be difficult to handle. In the second view, ${ }^{7}$ it has been expressed that if there were some way of learning the phases, then it would be a routine matter to find the arrangement of atoms in any given crystal no matter how complicated.

Recently, a similar independent work has also been carried out by Werner et $a l .^{9}$

The author is very grateful to Professor C. N. Yang for his interest in the present work, and to Professor R. Colella for interesting discussions on the crystal diffraction of coherent $\mathrm{x}$-ray and neutron beams. Thanks go to Professor J. J. Broderick for reading the manuscript. 
${ }^{1}$ Ming Chiang Li, Phys. Rev. A 10, 781 (1974); Phys. Rev. B 12, 3150 (1975).

${ }^{2}$ Ming Chiang Li, Phys. Rev. A $\underline{9}, 1635$ (1974); 11, 1101 (1975).

${ }^{3}$ L. Marton, Phys. Rev. 85, 1057 (1962); L. Marton, J. A. Simpson, and J. A. Suddeth, Rev. Sci. Instrum. 25, 1099 (1954).

${ }^{4} \mathrm{U}$. Bonse and M. Hart, Appl. Phys. Lett. 6, 155 (1965).

${ }^{5} \mathrm{H}$. Rauch, W. Treimer, and U. Bonse, Phys. Lett. A 47, 369 (1974).

${ }^{6}$ L. V. Azaroff, Elements of X-ray Crystallography (McGraw-Hill, New York, 1966); U. Bonse and M. Hart, Z. Phys. 190, 455 (1966); R. Colella, Phys. Rev. B 6, 4857 (1972).

${ }^{7}$ M. J. Buerger, Crystal-Structure Analysis (Wiley,
New York, 1960).

${ }^{8} \mathrm{~J}$. M. Cowley, Diffraction Physics (North-Holland, Amsterdain, 1975).

${ }^{9}$ S. A. Werner, R. Colella, A. W. Overhauser, and C. F. Eagen, in Proceedings of the International Conference on Neutron Scattering, Gatlinburg, Tennessee, 1976 (unpublished). In their scheme all incident and diffracted beams are confined in a single plane. The alignment is accomplished by adjusting the incident neutron wavelength. The alignment procedure discussed in the present manuscript is more general. It shows in a simple example that, by relaxing the single plane confinement, the alignment can be achieved even without adjusting the incident neutron wavelength. 